\title{
Civilian Surge Key to Complex Operations
}

\author{
Edited by \\ Hans Binnendijk and \\ Patrick M. Cronin
}

Published for the Center for Technology and National Security Policy by National Defense University Press

Washington, D.C.

2009 